\title{
Una actualización en el tratamiento de la artritis reumatoide en pacientes con factores de mal pronóstico
}

\author{
Najany Andrea Ibarra Alvarado*, Leslie Elizabeth Leiva Rodríguez*, Diego Issac Tiscareño Flores*, Giselle \\ Adriana Torres Hernández*, Terrones-Saldivar Ma del Carmen**
}

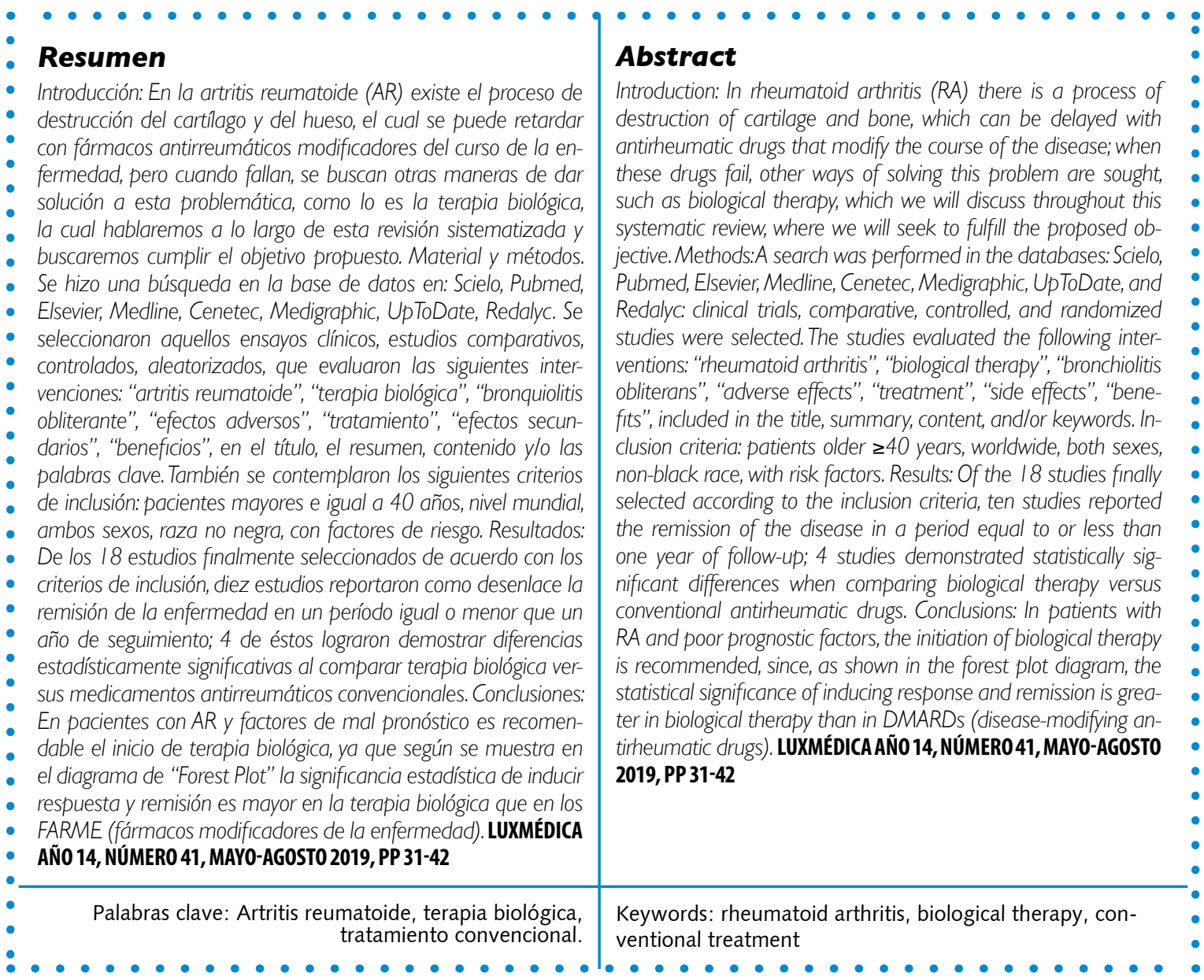

* Estudiantes del octavo semestre de la carrera de Medicina del Centro de Ciencias de la Salud de la Universidad Autónoma de Aguascalientes ** Profesora Investigadora de Tiempo Completo del Centro de Ciencias de la Salud de la Universidad Autónoma de Aguascalientes.

Fecha de recibido: 27 de noviembre 2019

Fecha de aceptado: 19 de marzo 2019

Correspondencia: Dra en C Ma del Carmen Terrones Saldívar. Edificio 101, planta alta. Centro de Ciencias de la Salud. Universidad Autónoma de Aguascalientes. Avenida Universidad 940. Código postal 20131. Teléfono (449) 9108433. Correo electrónico mcterron@correo.uaa.mx 


\section{Introducción}

La artritis reumatoide (AR) es una enfermedad inflamatoria, crónica, autoinmune y sistémica de etiología desconocida; su principal órgano blanco es la membrana sinovial; se caracteriza por inflamación poliarticular y simétrica de articulaciones grandes y pequeñas, con potencial compromiso sistémico en cualquier etapa de su evolución. ${ }^{1}$

Actualmente, se desconoce la causa exacta de la AR. Existe un elemento genético que, cuando se combina con un "desencadenante" ambiental como virus, infección, estrés o trauma, puede causar un mal funcionamiento del sistema inmunológico. Fumar cigarrillos es un factor precipitante importante (y también empeora las perspectivas de la AR). En la actualidad no hay cura. ${ }^{2-4}$

La AR afecta del 0.2 al $2 \%$ de la población mundial, principalmente al grupo etario con mayor capacidad laboral o productiva dentro de la sociedad. La edad de inicio es a los 40 años \pm 10 años (25-50 años, aunque puede comenzar a cualquier edad). Es más frecuente en mujeres que en hombres, con una relación de 3:1, esta diferencia entre sexos disminuye a edades más avanzadas. ${ }^{5-6}$

Si bien, aproximadamente del 5 al 20\% de los pacientes con AR presentan un curso monocíclico o autolimitado, el resto de los pacientes presentan patrones de curso policíclico con exacerbaciones y remisiones que van desde parciales hasta totales o de curso rápidamente progresivo, que de no limitarse ocasionarían daño articular irreversible, limitación funcional y discapacidad así como disminución en la calidad de vida de los pacientes. ${ }^{1-3,7-10}$

La AR es un problema de salud pública en Estados Unidos de América y países desarrollados; por su prevalencia, las consecuencias funcionales, el impacto económico y el incremento de los servicios de salud, sólo en Estados Unidos de Norteamérica genera aproximadamente 9 millones de visitas médicas y 250,000 hospitalizaciones anuales, una pérdida de 17,6 billones en salarios y una invalidez permanente de $2.5 \%$ por año. La mortalidad reportada en pacientes con $A R$ es mayor que en la población general con una tasa estandarizada de 2.26 , demostrándose una reducción en su expectativa de vida. ${ }^{1-3}$

La buena noticia es que el pronóstico actual, si la AR se diagnostica y trata a tiempo, es significativamente mejor que hace 20 o 30 años. Muchas personas tienen una mejor calidad de vida a pesar de tener AR. ${ }^{2}$

Actualmente el proceso de destrucción del cartílago y del hueso que se produce en la AR se puede retardar con la administración de un grupo de fármacos distintos conocidos como Fármacos antirreumáticos modificadores del curso de la enfermedad (FARME), incluyendo: antipalúdicos (cloroquina, hidroxicloroquina), sulfasalazina. penicilamina, inmunosupresores (azatioprina, ciclofosfamida, metotrexato) y sales de oro. Estos fármacos no producen una mejoría inmediata, pero requieren de 4 a 6 meses de tratamiento para una 


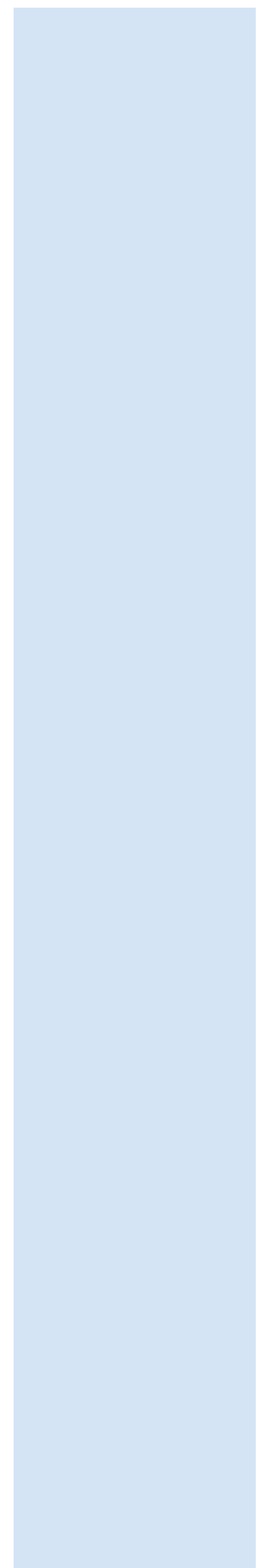

respuesta completa. El uso a largo plazo se limita por su toxicidad y pérdida de eficacia. Si un fármaco no demuestra beneficio en 6 meses, tiene que retirarse y sustituirse por otro FARME. ${ }^{3-4,8-14}$

En este orden de ideas, el manejo de la AR ha cambiado drásticamente en los últimos 30 años. En la década de 1980, se descubrió que las articulaciones con inflamación activa de las personas con AR contienen muchos químicos diferentes que causan inflamación o que llegan a contribuir a ello, producido por células en la articulación. Entre estas sustancias químicas se descubrió que muchas eran proteínas llamadas citoquinas, cuyo trabajo es enviar mensajes químicos de una célula a otra. Hay muchas citoquinas diferentes: algunas disminuyen la inflamación y otras son particularmente potentes para causarla. ${ }^{3-4}$

Es de esta forma que se empieza a explorar el campo de los agentes biológicos en reumatología. Los medicamentos biológicos para el tratamiento de la AR están hechos de proteínas. Funcionan al bloquear la actividad de un químico o célula clave involucrado en la inflamación que da lugar a la inflamación de las articulaciones y otros síntomas. Son terapias potentes y específicas. Actualmente, se dispone de ocho agentes biológicos para el tratamiento de la AR: cinco medicamentos que actúan bloqueando el factor de necrosis tumoral alfa (anti-TNF): infliximab, etanercept, adalimumab, certolizumab y golimumab; un inhibidor de la IL-1: anakinra; una proteína de fusión moduladora de la activación de células T: abatacept y un anticuerpo monoclonal contra el receptor de la IL-6: tocilizumab. ${ }^{3-4,18}$

En la actualidad se encuentran pacientes desde aquellos que asisten a consulta externa hasta aquellos que llegan a la sala de urgencias a causa de alguna de sus complicaciones, por lo que representa un fuerte problema para salud pública, los recursos económicos del hospital y un deterioro en la calidad de vida para el paciente. Por lo anterior expuesto, nos proponemos entonces a realizar un análisis detallado acerca del manejo de la AR y la prevención de sus complicaciones. El objetivo de este trabajo fue establecer marcos de referencia para la práctica clínica que sean capaces de guiar, orientar e ilustrar las decisiones del personal de salud para lograr una mejoría en la efectividad, seguridad y calidad de la atención en los individuos que padecen AR asociada a factores de mal pronóstico para la enfermedad, para su oportuno, individualizado y correcto tratamiento farmacológico, ya sea, con fármacos modificadores de la enfermedad o el uso de la terapia biológica.

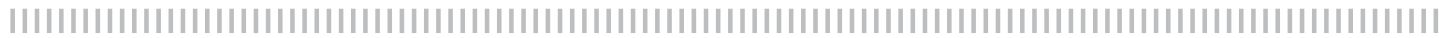

\section{Material y Métodos}

Tipo de publicación: revisión sistematizada de revistas científicas online.

Protocolo de búsqueda y selección de los estudios: se aplicaron las fases de identi- ficación, tamización, elección e inclusión de la guía PRISMA (Preferred Reporting Items for Systematic Reviews and MetaAnalyses). 
Búsqueda e identificación de los estudios: se hizo una búsqueda en la base de datos en: Scielo, Pubmed, Elsevier, Medline, Cenetec, Medigraphic, UpToDate, Redalyc. Las sintaxis empleadas fueron: Pubmed, Elsevier, Scielo, Medline, SciencieDirect: ((AR)) AND ((terapia biológica)) AND ((bronquiolitis obliterante)) AND ((efectos adversos)) AND ((tratamiento)) AND ((protocolo de valoración para terapia biológica)). Medigraphic, UpToDate, Redalyc. org: ((AR)) AND ((terapia biológica)) AND ((efectos secundarios)) AND ((actualizaciones en el tratamiento)) AND ((beneficios)). Se utilizó el idioma español (principalmente) y el inglés. El algoritmo de tamizaje se puede apreciar en la figura 1.

Intervalo de tiempo para la obtención de los documentos fue de 1984-2018.

\section{Criterios de inclusión:}

- Ensayos clínicos, estudios comparativos, controlados, aleatorizados, que evaluaron las siguientes intervenciones: "AR", "terapia biológica", "bronquiolitis obliterante", "efectos adversos", "tratamiento", "protocolo de valora- ción para terapia biológica", "efectos secundarios", "beneficios", "actualizaciones en el tratamiento", en el título, el resumen, contenido y/o las palabras clave.

- Pacientes mayores e igual a 40 años, a nivel mundial.

- Ambos sexos.

- Raza no negra.

- Con factores de riesgo (fumadores y no fumadores, con enfermedad pulmonar intersticial, historia familiar, infecciones). ${ }^{16-19}$

Criterios de exclusión: De los artículos seleccionados se excluyeron los siguientes:

- Edad era menor a 40 años.

- Raza negra.

- Tamaño de muestra inferior a 10.

- Artículos no disponibles en las bases de datos.

- Uso previo de agentes biológicos.

- Pacientes embarazadas o en etapa de lactancia.

- Pacientes en terapia biológica por enfermedades reumatológicas diferentes a la AR.

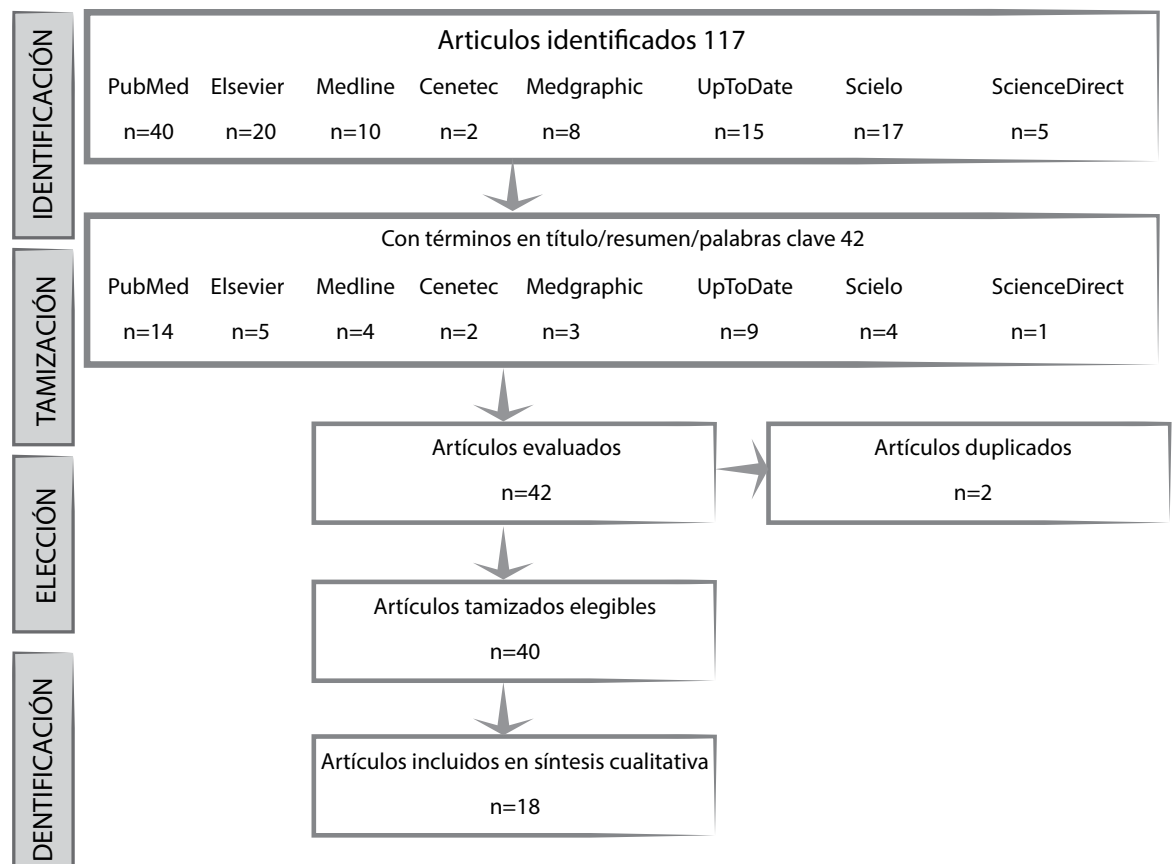


Análisis de reproducibilidad y evaluación de la calidad metodológica. Tipo de análisis utilizados: variabilidad, fiabilidad y validez.
Elaboración de gráficos: para la realización del diagrama de Forest Plot se utilizó el programa MedCalc ${ }^{\circledR}$ versión 13.3.3.0 copyright (C) 1993-2014 bajo la licencia de Team EAT 2014.

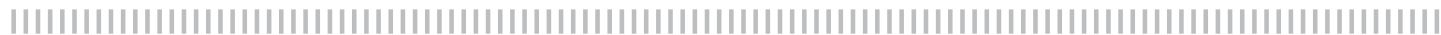

\section{Resultados}

La revisión sistemática de la literatura identificó 117 estudios potencialmente relevantes (Pubmed: 40; Elsevier: 20; Medline: 10; Cenetec: 2, Medigraphic: 8, UptoDate: 15, Scielo: 17; SciencieDirect: 5) de los cuales 45 fueron escogidos para la evaluación completa en pro de evaluar la elegibilidad.

Fueron excluidos 2 artículos duplicados, porque no cumplían con el criterio establecido para el tiempo de duración de la enfermedad, correspondiente a menos de un año en AR temprano, adicionalmente, a que la metodología del estudio no correspondía a ensayos clínicos o estudios (comparativos, controlados o aleatorizados). En la evaluación para realizar la síntesis cualitativa y cuantitativa se incluyeron un total de 18 estudios (fig. 1). Las características de los estudios incluidos son descritas de forma completa en la tabla 1 de la presente revisión.

Los resultados relacionados con la remisión clínica; en pacientes con características similares a nuestro sujeto de prueba, fueron reportados en la mayoría de los estudios según el puntaje en la escala DAS28, considerándose remisión un puntaje menor de 2,6.

Diez estudios concluyeron en la remisión de la enfermedad en un período $<1$ año de seguimiento; 4 de ellos demostraron diferencias estadísticamente significativas al comparar terapia biológica contra fármacos antirreumáticos convencionales. HørslevPetersen y cols. encontraron una mayor proporción de pacientes con DAS28 $<2,6$ a los 12 meses en el grupo de adalimumab/
MTX/corticoide intraarticular comparado con $M T X /$ corticoide intraarticular, siendo esta del 74 y $49 \%$, respectivamente, con un $R R=1,51$ (IC 95\%: 1,18-1,93; $p=$ 0,001). En el grupo estudiado por Takeuchi y cols., se evidenció que los pacientes tratados con adalimumab más MTX tuvieron mayor probabilidad de alcanzar remisión clínica (31\%) comparados con el grupo asignado a MTX en monoterapia (14,5\%) a la semana 26 de tratamiento, con un RR $=2,11$ (IC 95\%: 1,37-3,24; $p=0,007$ ). Adicionalmente, el estudio conducido por Emery y cols. mostró un mayor número de pacientes con remisión clínica a las 52 semanas en el grupo tratado con etanercept/ MTX $(50 \%)$. En relación con los pacientes tratados con MTX en monoterapia (28\%), con un $R R=1,79$ (IC 95\%: 1,43-2,26; $p$ $=0,00001)$. Snitch y cols. encontraron una mayor proporción de pacientes con remisión clínica a la semana 12 de tratamiento en el grupo tratado con abatacept/MTX $(50 \%)$. En relación con los pacientes tratados con MTX en monoterapia (20\%), con un $\mathrm{RR}=4.68$ (IC 95\%: 1,18-18,6; $p=0,00001) \cdot{ }^{20-28}$

Cinco estudios evaluaron la remisión de la AR en pacientes que tuvieron seguimiento durante 13 y 24 meses posteriores al inicio de tratamiento, mostrando diferencias significativas el estudio realizado por O'Dell y cols., el cual sustenta el uso de MTX en monoterapia como manejo inicial de pacientes con AR con factores de mal pronóstico, logrando remisión en el $50,6 \%$ de los pacientes tratados con MTX comparado con el $34,2 \%$ de pacientes que recibieron terapia combinada progresiva 
(MTX + etanercept o MTX + sulfasalazina + hidroxicloroquina) (RR $=0,67$ [IC 95\%: $0,51-0,88] ; p=0,004) .{ }^{29-33}$

Tres estudios, Yvonne y cols. $(\mathrm{RR}=$ 1,01 [IC 95\%: 0,76-1,33]; $p=0,001$ ); Yamanaka y cols. (RR $=1,01$ [IC 95\%: $0,71-1,45] ; p=0,004) ;$ Martín Soubrie y cols. (RR $=0,66$ [IC 95\%: 0,4-1,11]; $p$ $=0,01$ ); no obtuvieron inclinación alguna por la terapia convencional sobre la terapia biológica, en la remisión de AR en un lapso de 13 a 24 meses. ${ }^{34-37}$

Se realizó una evaluación del riesgo de sesgos teniendo en cuenta los siguientes aspectos: la presencia de sesgo de selección, medida por la generación de la secuencia y el ocultamiento de la asignación; presencia de sesgo de realización, evaluando el cegamiento de los participantes y del personal; existencia de sesgo de detección, si no presentaban cegamiento de evaluadores del resultado y sesgo de desgaste, relacionado con datos de resultados incompletos (pérdida de datos/pacientes mayor del $10 \%$ de los incluidos al inicio del estudio). En forma general, se encontró un alto riesgo de sesgos.

\section{Tabla |}

\section{Datos empleados en el diagrama de Forest Plot}

\begin{tabular}{|c|c|c|c|}
\hline STUDY & ODDS_RATIO & LOW & HIGH \\
\hline Lampropoulos CE et. al. 2015 & 1.2 & 23.1 & 34.7 \\
\hline Salas Mena, et al. 2015 & 1.98 & 1.64 & 2.39 \\
\hline Snitch et. al. 2009 & 4.68 & 1.18 & 18.6 \\
\hline Emery, et al. 2017 & 1.79 & 1.43 & 2.26 \\
\hline Yvonne et al.,2007 & 1.01 & 0.76 & 1.33 \\
\hline Yamanaka et al.,2014 & 1.01 & 0.71 & 1.45 \\
\hline Martin Soubrie et al.,2009 & 0.66 & 0.4 & 1.11 \\
\hline Goekoop-Ruiterman et al.,200 & 1 & 0.75 & 1.34 \\
\hline Hørslev-Petersen et al.,2013 & 1.51 & 1.18 & 1.93 \\
\hline Takeuchi et al.,2014 & 2.11 & 1.37 & 3.24 \\
\hline Bejarano et al.,2010 & 1.87 & 0.85 & 4.11 \\
\hline Breedveld et al. 2008 & 1.03 & 0.84 & 1.28 \\
\hline Leirisalo-Repo et al.,2013 & 0.84 & 0.62 & 1.14 \\
\hline Nam et al.,2014 & 1.13 & 0.78 & 1.65 \\
\hline Juhász et al.,2014 & 1.06 & 0.85 & 1.32 \\
\hline DurezP et al.,2007 & 0.96 & 0.8 & 1.15 \\
\hline Detert et al.,2013 & 0.93 & 0.54 & 1.96 \\
\hline O'Delletal.,2013 & 0.67 & 0.51 & 0.88 \\
\hline
\end{tabular}

Lampropoulos CE et al 2015 Salas Mena et al 2015 Snitch et al 2009 Emery et al 2017

Yvonne et al 2007

Yamanakaetal 2014 Martin Soubrie et al 2009 Goekoop-Ruiterman et al 200 Horslev-Petersen et al 2013

Takeuchi et al 2014 Bejarano et al 2010

Breedveld et al 2008 Leirisalo-Repo et al 2013

Nam et al 2014

O’Delletal 2013

Detert et al 2013 DurezP et al 2007

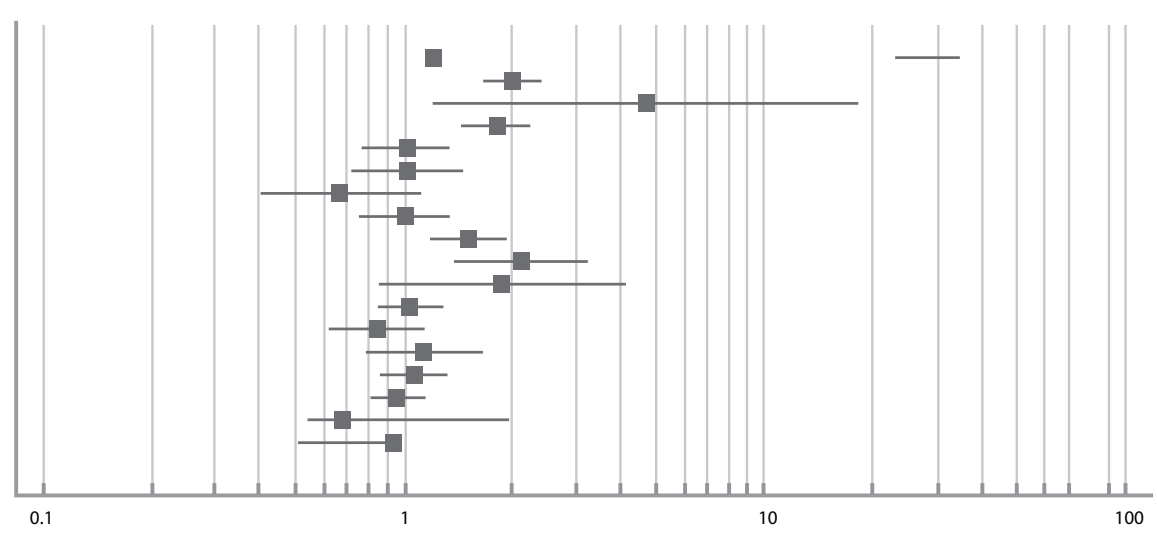

Fig. No. 2 diagrama de Forest Plot 


\section{Tabla 2}

Datos de los artículos seleccionados

EPO: Enfermedad Pulmonar Obstructiva. AR: Artritis Reumatoide. BO: Bronquiolitis Obliterante. EPI: Enfermedad Pulmonar Intersticial. b-FARME: Fármaco antirreumático biológico modificador de la enfermedad.

\begin{tabular}{|c|c|c|c|c|c|c|c|c|}
\hline & AÑo & PAís & N & Descripción & Nombre & ODDS & Límite & Límite \\
\hline 1 & 1984 & España & 10 & AR asociada a EPO & - & - & - & - \\
\hline 2 & 2008 & México & & Artículo de Revisión AR y Pulmón. & - & - & - & - \\
\hline 3 & 1980 & España & 10 & $\begin{array}{l}\text { Bronquiolitis Obliterante con Neumonía } \\
\text { Organizada en paciente con AR }\end{array}$ & - & - & - & - \\
\hline 4 & 2010 & México & $\ldots \ldots .$. & Tratamiento en pacientes con AR & - & - & - & - \\
\hline 5 & 1988 & España & 10 & BO en pacientes con AR & - & - & - & - \\
\hline 6 & 2015 & México & $\ldots . .$. & Tratamiento de EPI en pacientes con AR & - & - & - & - \\
\hline 7 & 2018 & España & 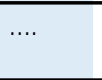 & $\begin{array}{l}\text { IL-6 en AR Tratamiento Actual de la } \\
\text { afectación pulmonar }\end{array}$ & - & - & - & - \\
\hline 8 & 2010 & Argentina & $\ldots$ & Revisión Sistemática & - & - & - & - \\
\hline 9 & 1987 & Inglaterra & 112 & Resultados a largo plazo de la AR & - & - & - & - \\
\hline 10 & & & & & $--\ldots \ldots$ & $-\ldots \ldots$. & $-\ldots .$. & $-\ldots .$. \\
\hline 11 & 2003 & USA & 1529 & Infecciones como complicación de AR & - & - & - & - \\
\hline 12 & 2013 & Inglaterra & 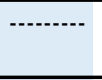 & $\begin{array}{l}\text { Revisión sistemática de la Guía de } \\
\text { terapias biológicas en AR }\end{array}$ & - & - & - & - \\
\hline 13 & 2016 & USA & 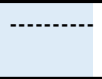 & $\begin{array}{l}\text { Revisión sistemática Recomendaciones } \\
\text { para el manejo de AR con terapia biológica }\end{array}$ & - & - & - & - \\
\hline 14 & 2012 & USA & 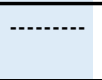 & $\begin{array}{l}\text { Revisión sistemática del uso de la terapia } \\
\text { biológica en } A R\end{array}$ & - & - & - & - \\
\hline 15 & 2011 & CostaRica & 47 & $\begin{array}{l}\text { Respuesta clínica a la terapia anti-TNF } \alpha \\
\text { en pacientes con AR con falla o intole- } \\
\text { rancia a FARME no biológicos }\end{array}$ & $\begin{array}{l}\text { Respuesta clínica a la } \\
\text { terapia anti-TNF } \alpha \text { en } \\
\text { pacientes con AR con } \\
\text { falla o intolerancia a } \\
\text { FARME no biológicos }\end{array}$ & 1.2 & 23.1 & 34.7 \\
\hline 16 & 2011 & México & $-\cdots-\cdot$ & Diagnóstico y tratamiento en AR en adultos & - & - & - & - \\
\hline 17 & 2018 & Alemania & $\begin{array}{ll}-\cdots--\cdot \\
-\cdots\end{array}$ & Niveles séricos de agentes biológicos en AR & - & - & - & - \\
\hline 18 & 2011 & España & $\begin{array}{l}-\cdots- \\
-\cdot-\end{array}$ & Terapias biológicas en el tratamiento de AR & - & - & - & - \\
\hline 19 & 2015 & Grecia & 1403 & $\begin{array}{l}\text { Eventos adversos e infecciones en pacientes } \\
\text { tratados con terapia biológica }\end{array}$ & $\begin{array}{l}\text { Eventos adversos e in- } \\
\text { fecciones en pacientes } \\
\text { tratados con terapia } \\
\text { biológica }\end{array}$ & 1.98 & 1.64 & 2.39 \\
\hline 20 & 2004 & $\begin{array}{l}\text { Madrid, } \\
\text { España }\end{array}$ & 576 & Rol de los FARME & - & - & - & - \\
\hline 21 & 2013 & $\begin{array}{l}\text { Madrid, } \\
\text { España }\end{array}$ & $\cdots$ & $\begin{array}{l}\text { Revisión sistemática sobre nuevas } \\
\text { estrategias en el tratamiento de AR }\end{array}$ & - & - & - & - \\
\hline 22 & 2014 & $\begin{array}{l}\text { Copenague } \\
\text { Dinamarca }\end{array}$ & 107 & $\begin{array}{l}\text { Preferencias de los pacientes y los } \\
\text { médicos en la admin. de b-FARME }\end{array}$ & - & - & - & - \\
\hline 23 & 2015 & EUA & 89 & Relación de la AR con la bronquiolitis obliterante & - & -- & - & - \\
\hline 24 & 2004 & $\begin{array}{l}\text { Londres, } \\
\text { Inglaterra }\end{array}$ & 85 & Relación de la AR con la bronquiolitis obliterante & - & - & - & - \\
\hline 25 & 2004 & Alemania & $\cdots$ & $\begin{array}{l}\text { Reporte de caso de bronquiolitis obliterante } \\
\text { en un paciente con AR tratado con sulfazalacina }\end{array}$ & - & - & - & - \\
\hline 26 & 2011 & & $\cdots$ & $\begin{array}{l}\text { Revisión sistemática: eficacia del tratamiento } \\
\text { con b-FARME }\end{array}$ & 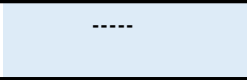 & $\cdots-\cdot$ & 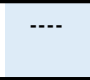 & $\cdots$ \\
\hline 27 & 2016 & Japón & 36,504 & $\begin{array}{l}\text { Predictores en el inicio de la terapia biológica } \\
\text { en hospitales de Japón }\end{array}$ & $\cdots-\cdot$ & $\cdots$ & $\cdots$ & $\cdots$ \\
\hline
\end{tabular}




\begin{tabular}{|c|c|c|c|c|c|c|c|c|}
\hline 28 & 2013 & EUA & 8,037 & $\begin{array}{l}\text { Comparación de los efectos de los b-FARME } \\
\text { en pacientes con AR }\end{array}$ & - & - & - & - \\
\hline 29 & 2011 & España & $\cdots$ & Guía de satisfacción de la terapia biológica & $-\cdots-$ & $\cdots$ & ---- & --- \\
\hline 30 & 2015 & Ecuador & $\cdots$ & $\begin{array}{l}\text { Comparación de la eficacia terapéutica } \\
\text { entre infliximab adalimumab y } \\
\text { etanercept, en el tratamiento de AR }\end{array}$ & $\cdots$ & - & - & - \\
\hline 31 & 2013 & México & 4750 & Terapias biológicas en AR & - & - & - & - \\
\hline 32 & 2012 & España & $\cdots$ & Cambios en el curso de la AR con terapia biológica & - & - & - & - \\
\hline 33 & 2013 & México & $\cdots$ & Terapia biológica en la AR & - & 4,68 & 1.18 & 18.6 \\
\hline 34 & 2008 & México & - & Terapéutica en la AR & - & - & - & - \\
\hline 35 & - & - & - & - & - & - & - & - \\
\hline 36 & 2008 & Colombia & 1726 & Terapia biológica en AR temprana & - & 4.0 & 1.43 & 2.26 \\
\hline 37 & 2011 & Cuba & 24 & $\begin{array}{l}\text { Beneficios clínicos después de haber } \\
\text { tratado con itolizumab }\end{array}$ & - & - & - & - \\
\hline 38 & 2009 & Colombia & 31 & Beneficios en pacientes tras recibir infliximab & - & - & - & - \\
\hline 39 & 2014 & España & - & Avances en AR & - & - & - & - \\
\hline
\end{tabular}

\section{Discusión}

Como toda enfermedad, la AR requiere un abordaje integral con intervenciones no farmacológicas (cambios en el estilo de vida, ejercicios físicos, terapia ocupacional e interrupción del tabaquismo además del uso FARME, que incluyen el metotrexate (MTX), antimaláricos (hidroxicloroquina, cloroquina), leflunomida, sulfasalazina. En la actualidad se usan con mayor frecuencia nuevos grupos de medicamentos que, como la terapia biológica o b-FARME biológicos (anti-TNF y no anti- TNF), han empezado a demostrar un papel importante como modificadores del curso clínico de la AR y la remisión de la misma. Las estrategias de manejo en AR han cambiado significativamente en los últimos años en pro de disminuir la carga sintomática de la enfermedad, la progresión del daño articular y la discapacidad funcional. 37-39

El estudio realizado por Takeuchi y cols., demostraron que el agente anti-TNF, adalimumab, fue eficaz en el tratamiento de la AR, y los resultados de un subanálisis del estudio DEO19 indican que el tratamiento temprano con adalimumab puede ser más eficaz que su uso tardío en el curso de la AR. ${ }^{15}$

En el artículo de la "Respuesta clínica a la terapia anti-TNF $\alpha$ en pacientes con AR con falla o intolerancia a FARME no biológicos en el Hospital San Juan de Dios en el periodo de enero del 2006 a diciembre de 2011". El estudio ha sido descriptivo, retrospectivo, observacional, mediante la revisión de expedientes de pacientes con AR que iniciaran terapia anti-TNF $\alpha$ donde se ha valorado el DAS28 y VES inicial, luego a los 6 meses y 12 meses después de inicio del tratamiento. En el periodo analizado, de 47 pacientes evaluados con falla a FAR$M E$ tradicionales que han recibido terapia anti-TNF $\alpha$, según DAS28, 32 pacientes fueron catalogados como actividad severa y 15 de ellos con actividad moderada. Un total de 41 pacientes han utilizado etanercept, 6 pacientes adalimumab. El DAS28 promedio inicial fue de 5,63 en todos los pacientes, de 6,16 en el subgrupo de pacientes con actividad severa, y de 4,49 en el subgrupo de actividad moderada. Luego de 6 meses de tratamiento, el DAS28 ha descendido a 3,25 en todos los pacientes, con un 3,67 en el subgrupo de actividad clínica severa, y un 2,35 en el subgrupo de actividad clínica moderada. Al año de tratamiento, los valores de DAS28 han sido 3,13 para todos los pacientes, 3,53 en los de actividad severa y 2,28 en el subgrupo de actividad clínica moderada. En todos los 
grupos y subgrupos de pacientes fue demostrado diferencia estadísticamente significativa con $p$ menor de 0,05. La terapia anti-TNF $\alpha$ ha demostrado ser efectiva para mejoría de actividad clínica de la $A R$, independientemente de la severidad de base $y$ del tipo de terapia anti-TNF $\alpha$ empleada. ${ }^{15}$

El artículo de "Cambios inducidos en DKK1 en pacientes con AR que inician tratamiento con terapia biológica" es un estudio por cohortes, prospectivo de pacientes con diagnóstico de AR activa evaluados en la Unidad de Reumatología, y que inician terapia biológica. Para el diagnóstico de AR se aplicarán los criterios ACR 1987. Los criterios de inclusión fueron los siguientes: diagnóstico de $A R$, edad mayor de 18 años, presencia de actividad de la enfermedad (DAS $>2,4$ ) a pesar de tratamiento con fármacos modificadores sintéticos de la enfermedad, y firma de consentimiento informado. Se excluyeron pacientes con eventos cardiovasculares previos, fracturas osteoporóticas previas, enfermedad metabólica ósea distinta a osteoporosis, enfermedad renal crónica, hepatopatía crónica, diabetes mellitus tipo 1 y 2 , enfermedad neoplásica, embarazo y lactancia. Nuestro estudio mostró una disminución en los niveles de DKK-1 a los seis meses de tratamiento, lo que está en concordancia con lo publicado recientemente por Briot y cols. Podemos decir que en pacientes con AR activa tratados con terapia biológica han observado un descenso no significativo de las concentraciones séricas de DKK1 y un aumento significativo de la resorción. ${ }^{34}$

En el artículo de revisión de la "Terapia biológica en la AR temprana: eficacia en la remisión de la enfermedad. revisión sistemática de la literatura". Se hizo una búsqueda sistematizada de la literatura en bases de datos electrónicas especializadas en ciencias de la salud: PubMed, Embase, Cochrane, LILACS. Criterios de inclusión: pacientes mayores de 18 años con AR (curso clínico menor de 12 meses) según criterios del Colegio Americano de Reumatología/ Liga Europea contra el Reumatismo (ACR 1987, ACR/EULAR 2010), que recibieron terapia biológica en monoterapia o combinada con otros fármacos antirreumáticos modificadores de la enfermedad (FARME) y fueron incluidos en estudios clínicos controlados aleatorizados. Hay diferencias a favor en el desenlace de remisión de la enfermedad en pacientes con AR que reciben terapia biológica versus pacientes tratados con FARME convencionales. ${ }^{37}$

En contraste, en el caso de "Eventos adversos e infecciones en pacientes con $A R$ tratados con fármacos convencionales 0 agentes biológicos: un estudio real". Donde se realizó un análisis de las historias clínicas de los pacientes seguidos en una sola clínica ambulatoria. En total, 1403 adultos (295 hombres, 1108 mujeres) se incluyeron en el análisis (969 tratados solo con FARME sintéticos, 434 con terapia biológica). Todos los efectos adversos e infecciones se registraron y su gravedad se clasificó de acuerdo con los criterios internacionales. Se calcularon las tasas de incidentes y se realizaron parcelas de Kaplan-Meier y modelos de riesgos proporcionales de Cox para examinar la asociación de los grupos de tratamiento con el riesgo de cualquier efecto adverso. ${ }^{7}$

El riesgo de cualquier efecto adverso, independientemente de la gravedad, fue significativamente mayor en los pacientes con terapia biológica con un índice de riesgo ajustado de 1,98 (IC del 95\%: 1,64 a 2,39 ). Los pacientes en el grupo biológico tratados inicialmente con infliximab o adalimumab tuvieron un mayor riesgo de efectos adversos en comparación con los pacientes que recibieron etanercept u otros agentes biológicos. Entre los pacientes tratados con metotrexato, los que recibieron una dosis inferior a $10 \mathrm{mg}$ tuvieron un mayor riesgo de $A E$ en comparación con los que recibieron dosis más altas. ${ }^{20}$ 


\section{Limitaciones}

Las limitaciones se debieron, principalmente, por la heterogeneidad de los estudios por diferencias clínicas y epidemiológicas. En el presente estudio se demostró que existe una diversidad significativa entre los estudios meta-analizados, así como un alto riesgo de sesgos demostrado a través del sistema GRADE. Esta heterogeneidad radicó en diferencias en el diseño de los estudios analizados, destacando aquellas en el comparador (por ej., en algunos estudios fue MTX monoterapia mientras que en otros fueron terapias combinadas); de igual manera en el tiempo de medición de desenlace, el cual fue variable entre todos los estudios (proporción en remisión a los $6,12,24$ o 60 meses), entre otras.

Existen limitaciones al evaluar la remisión de la enfermedad mediante el DAS28 debido a que un importante grupo de pacientes, que son clasificados en remisión por medio de esta herramienta, no presentaban factores de mal pronóstico correspondiente a un alto nivel de actividad de la AR.

Todas estas limitaciones invitan al lector a analizar con cautela los resultados del estudio en el momento de llevarlos a la práctica clínica.

\section{Conclusiones}

En el Diagrama de Bosque, los fármacos que tienen mayor probabilidad estadísticamente significativa de inducir respuesta y remisión serían adalimumab y etanercept, aunque los estudios que los soportan tienen limitaciones metodológicas.

Al evaluar esta revisión sistemática se llegó a la conclusión de que en pacientes con AR y factores de mal pronóstico (erosiones radiográficas, manifestaciones extraarticulares y multisistémicas, entre otros) es recomendable el inicio de terapia biológica, ya que según se muestra en el diagrama de "Forest Plot" la significancia estadística de inducir respuesta y remisión es mayor en la terapia biológica que en los FARME.

En contraste, el manejo de pacientes que están exentos de los factores de mal pronóstico, su tratamiento debe basarse en evitar las deformidades y conservar una buena capacidad funcional. El tratamiento en estos pacientes consiste en ejercicios físicos para mantener la movilidad articular y evitar la atrofia muscular, una dieta correcta que ayude a disminuir la actividad inflamatoria que presentan estos pacientes. Además, debe añadirse una terapia farmacológica, que consiste en: analgésicos y AINE's para disminuir el dolor e inflamación, corticoides a dosis bajas por el riesgo de complicaciones dosis dependientes, y el uso de FARME para modificar el curso de la enfermedad.

\section{Conflicto de interés}

Ninguno de los autores declara conflicto de intereses para la publicación de este artículo. 


\section{Bibliografía}

1-. Instituto Mexicano del Seguro Social. Diagnóstico y Tratamiento de Artritis Reumatoide del Adulto. 2010. 7-97.

2-. Ailsa Bosworth. A Patient Guide to Biologic Therapies in the Treatment of Rheumatoid Arthritis. NRAS. 2013; 2-38.

3-. Recomendaciones EULAR para el tratamiento de la artritis reumatoide con fármacos antirreumáticos modificadores de la enfermedad sintéticos y biológicos: actualización de 2016. Ann Rheum Dis. 2017; 76: 960-977.

4-. Jasvinder A. Singh et al.2012 Actualización de las recomendaciones de 2008 del Colegio Americano de Reumatología para el uso de fármacos antirreumáticos modificadores de la enfermedad y agentes biológicos en el tratamiento de la artritis reumatoide. ACR AR. 2012; 64 (5): 625-639.

5-. Frederick Wolfe, Kaleb Michaud. Biologic Treatment of Rheumatoid Arthritis and the Risk of Malignancy. Arthritis \& Rheumatism. 2007; 56 (9): 2886-2895.

6-. Infections in Patients With Rheumatoid Arthritis Treated With Biologic Agents. Arthritis \& Rheumatism. 2005; 52 (11). 3403-3412.

7-. Lampropoulos CE, Orfanos P, Bournia VK, Karatsourakis $T$, Mavragani $C$, Pikazis $D$, Manoussakis $M N$, Tzioufas AG, Moutsopoulos HM, Vlachoyiannopoulos PG. Adverse events and infections in patients with rheumatoid arthritis treated with conventional drugs or biologic agents: a real world study. Epub. 2015;(2):16-24.

8-. Migliore A, Ballanti E, Laganà B, Martin LS, Frediani B. Biologic agents for rheumatoid arthritis: can we hypothesize new strategies of treatment?. Epub. 2014; (1):117-21.

9-. Huynh TK, Ostergaard A, Egsmose C, Madsen OR. Preferences of patients and health professionals for route and frequency of administration of biologic agents in the treatment of rheumatoid arthritis. Epub. 2014;(1):93-99.

10- Lin E, Limper AH, Moua T. Bronquiolitis obliterante asociada con artritis reumatoide: análisis de una serie de casos de un solo centro. BMC Pulm Med. 2018;18(1):105.

11- Martín-López M, Carmona L, Balsa A, Calvo-Alén J, Sanmartí R, Tornero J, Rosas J. Serum drug levels of biologic agents in the management of rheumatoid arthritis and spondyloarthritis: a systematic review. Epub. 2018;(15):22-28.

12- María Auxiliadora Castillo Muñoz, Ruth Ubago Pérez, Sandra Flores Moreno, Carmen Beltrán Calvo. Terapias biológicas en el tratamiento de la artritis reumatoide. eficacia y seguridad comparada entre diferentes agentes biológicos. 2011;(1):1-66.

13- Tvete IF, Natvig B, Gåsemyr J, Meland N, Røine $M$, Klemp M. Correction: Comparing Effects of Biologic Agents in Treating Patients with Rheumatoid Arthritis: A Multiple Treatment Comparison Regression Analysis. Epub. 2011;(1):1-5.

14- Rodríguez-Rodríguez $\mathrm{L}$, León $\mathrm{L}$, Ivorra-Cortes J, Gómez A, Lamas JR, Pato E, Jover JÁ, Abasolo L.
Treatment in rheumatoid arthritis and mortality risk in clinical practice: the role of biologic agents. Epub. 2016; (6):1026-1032.

15- Salas Mena, C. Respuesta clínica a la terapia antiTNF? en pacientes con artritis reumatoide con falla o intolerancia a FARME no biológicos en el Hospital San Juan de Dios en el periodo de enero del 2006 a diciembre del 2011. ETDEWEB. 2014;(2):80.

16- Bronquiolitis Obliterante En Una Paciente Afecta De Artritis Reumatoide. Arch Bronconeumol. 1985;21:127-130.

17- Edilzar Gilberto González Velásquez. Artritis Reumatoide y Pulmón. Neumología Y Cirugía De Tórax. 2008;67(2):75-78.

18-MP. Serrano Manero, B. Joven, R. Almodóvar, J. Balsalobre, PE. Carreira, I. Mateo. Bronquiolitis Obliterante con Neumonía Organizada en un Paciente con Artritis Reumatoide. Rev Esp Reumatol. 2003; 30 (8). 456-460.

19- Clínica Universitaria Facultad de Medicina Universidad de Navarra. Bronquiolitis Obliterante. 1991: 2935.

20-Mahlich J, Sruamsiri R. Treatment patterns of rheumatoid arthritis in Japanese hospitals and predictors of the initiation of biologic agents. Epub. 2016;(1):101-107.

21- Espiño Lorenzo $P$, et al. Comorbilidades iniciales en pacientes con artritis reumatoide a los que se les ha prescrito terapia biológica: un estudio de casos y controles. Clinica Reumatol. 2013; 9(1): 18-23.

22- Alejandro Muñoz Jiménez, Clara Aguilera Cros, Esteban Rubio Romero. La ciencia de la IL-6 en Artritis Reumatoide Tratamiento actual de la afectación pulmonar. Zanofy Genzyme. 2018;2-11.

23- José A. Maldonado Cocco. Agentes biológicos y manifestaciones extraarticulares de la artritis reumatoidea. Revista Argentina de Reumatología. 2010;21(3):32-36.

24- D.L. Scott, B.L. Coulton. D. PM. Symmons, A.J. Popert. Resultados A Largo Plazo Del Tratamiento De La Artritis Reumatoide: Resultados Después De 20 Años. ScienceDirect 1987; 329 (8542): 1108-1111.

25- Kaushik P, Cooper ES, Banda VR, Vatsavai SR, Kaushik R. Bronquiolitis obliterante con neumonía organizada en la artritis reumatoide: un caso fatal y breve reseña de la literatura. Reumatol Int. 2005; 25 (5): 390-391.

26- Uluba? B, Sahin G, Ozer C, Aydin O, Ozgür E, Apaydin D. Bronchiolitis obliterans organizing pneumonia associated with sulfasalazine in a patient with rheumatoid arthritis. Clin Rheumatol. 2004; 23(3):249-251.

27- Turkstra E, Ng SK, Scuffham PA. A mixed treatment comparison of the short-term efficacy of biologic disease modifying anti-rheumatic drugs in established rheumatoid arthritis. Curr Med Res Opin. 2011; 27 (10):1885-97.

28- Advira Health.Estudio De Satisfacción Con Las Terapias Biológicas, Desde La Visión De Los Pacientes Con Artritis Reumatoide. Conartritis. 2011; 1: 4-39.

29- Amay Sanchez Gabriel Natali. Comparación de la eficacia terapéutica entre infliximab adalimumab y 
etanercept, en el tratamiento de artritis reumatoidea en el IESS de Loja. 2015; 1: 4-62.

30- Guillermo Salinas-Escudero Juan Vargas-Valencia, Erika Gabriela García-García, Emilio Munciño-Ortega, Rosa María Galindo-Suárez. Etanercept y otras terapias biológicas en artritis reumatoide Un análisis de costo-efectividad. Rev Med Inst Mex Seguro Soc 2013; (5): 514-521.

31- Palma-Sánchez D, Haro-Martínez AC, Gallardo Muñoz I, Portero de la Torre M, Mayor González $M$ - Peñas E , Reyes-García R. Cambios inducidos en DKK1 en pacientes con artritis reumatoide que inician tratamiento con terapia biológica. Rev Osteoporos Metab Miner.2016; 8(1): 30-35

32-Manuel F. Ugarte-Gil, Eduardo M. Acevedo-Vásquez, Graciela S. Alarcón. Terapia biológica en enfermedades reumatológicas. Rev Med Hered. 2013; (24):141-155.

33- Lino E. Torres, Ariana Barbera y María del Carmen Domínguez. Principales estrategias terapéuticas en el tratamiento de la artritis reumatoide. CENIC. 2008; (39): 183-190.

34- Carmenza Beatriz Camargo Barrios, Jorge Eliécer
Rivas Ibargüena, Gerardo Quintana-López. Terapia Biológica En La Artritis Reumatoide Temprana: Eficacia En La Remisión De La Enfermedad. Rev Colomb Reumatol. 2017; 24(3):164-176.

35- Prada Hernández Dinorah $M$, et al. Artritis Reumatoide: beneficios clínicos observados en pacientes tratados con anticuerpo monoclonal Itolizumab. ( $T 1 \mathrm{~h} \mathrm{mAB}), 2$ años después de recibir tratamiento. Revista Cubana de Reumatología. 2011; 13 (17): 1-9.

36- John Londoño $\mathrm{P}$, et al. Cambio en la capacidad funcional, calidad de vida y actividad de la enfermedad, en un grupo de pacientes colombianos con artritis reumatoide refractaria al tratamiento convencional, que recibieron terapia con infliximab como medicamento de rescate. Rev. Med. 2009. 17 (1): 40-49.

37- Luis García Sevillano. Avances en artritis reumatoide. An. Real Acad. Farm. 2014; 80 (1): 126-150.

38- Cunnane $G$, Doran $M$, Bresnihan B. Infecciones y terapia biológica en la artritis reumatoide. Mejor práctica Res Clin Rheumatol. 2003; 17 (2): 345-363.

39-Sobre El Tratamiento De La Enfermedad Pulmonar Intersticial En Artritis Reumatoide. El Residente. 2015; 10 (3): 132-141. 